\title{
Ovaires et fertilité en 2019 : préservation de la fertilité, réserve ovarienne, ovaires polykystiques
}

Ovaries and fertility in 2019: fertility preservation, ovarian reserve, polycystic ovary syndrome

Sophie Catteau-Jonard ${ }^{\mathrm{a} * \mathrm{~b}, \mathrm{c}}$, Yves Ardaens ${ }^{\mathrm{d}}$, Didier Dewailly ${ }^{\mathrm{a}, \mathrm{b}, \mathrm{c}}$

${ }^{\text {a }}$ Service de gynécologie médicale, hôpital Jeanne-de-Flandre, CHRU de Lille, rue Eugène-Avinée, 59000 Lille, France

${ }^{\text {b }}$ Université de Lille, 42, rue Paul-Duez, 59000 Lille, France

${ }^{\mathrm{c}}$ Inserm U1172, place de Verdun, 59045 Lille, France

${ }^{\mathrm{d}}$ Cabinet de radiologie, 73, rue Jacquemars-Giélée, 59000 Lille, France

*Auteur correspondant : Sophie Catteau-Jonard ; e-mail : sophie.jonard@chru-lille.fr; tél. : +33-3 20 446309 ; fax : +33-320446407

Les auteurs n'ont pas de conflit d'intérêts à déclarer.

Titre court : Ovaires et fertilité en 2019 


\title{
Résumé
}

L'imagerie de la femme et en particulier l'échographie pelvienne jouent un rôle majeur dans

l'évaluation et le diagnostic d'anomalies de la réserve ovarienne, que ce soit dans les situations d'excès folliculaire tel que le syndrome des ovaires polykystiques, ou dans la situation inverse, c'est-àdire les baisses de réserve ovarienne, pouvant amener à proposer aux femmes une préservation de la fertilité.

\begin{abstract}
Women imaging, and in particular pelvic ultrasound, play a major role in the evaluation and diagnosis of ovarian reserve abnormalities. These situations are follicular excess such as polycystic ovary syndrome, or in the opposite situation, decreased ovarian reserve which may leads to offer fertility preservation.
\end{abstract}

\section{Mots clés}

Syndrome des ovaires polykystiques ; réserve ovarienne ; insuffisance ovarienne prématurée ; préservation de la fertilité

\section{Keywords}

Polycystic ovary syndrome; ovarian reserve; premature ovarian failure; fertility preservation

\section{Syndrome des ovaires polymicrokystiques}

\subsection{Physiopathologie}

Le syndrome des ovaires polymicrokystiques est la cause la plus fréquente d'anovulation, d'infécondité et d'hyperandrogénie chez la femme, puisqu'en effet, 5 à $10 \%$ des femmes en âge de procréer en sont affectées [1]. En dépit des efforts considérables pour en déterminer la cause, la physiopathologie du syndrome des ovaires polymicrokystiques reste mal comprise, mais les preuves s'accumulent pour suggérer que les principales anomalies sont primitivement ovariennes.

L'augmentation de la synthèse et de la sécrétion des androgènes par les ovaires est une caractéristique essentielle du syndrome des ovaires polymicrokystiques. Ce processus résulterait d'une dysrégulation intrinsèque des cellules stéroïdiennes de la thèque interne et pourrait secondairement être majorée par des facteurs ovariens tels que l'hormone lutéinisante (luteinizing hormone, LH) ou l'insuline. L'hyperandrogénie est en effet amplifiée par l'hyperinsulinisme qui augmente la fraction libre active des androgènes circulants en diminuant sa protéine de transport (sex hormone-binding globulin, SHBG) et qui accroît les effets de la LH sur la synthèse stéroïdienne des cellules thécales. 
Cette hyperandrogénie entraînerait également un excès de petits follicules en croissance (visible en échographie), probablement par un effet anti-apoptotique et un ralentissement de la cinétique folliculaire.

Enfin, il existe fréquemment dans le syndrome des ovaires polymicrokystiques un défaut de sélection du follicule dominant dû probablement à un inhibiteur de l'action de l'hormone folliculostimulante (follicle stimulating hormone, FSH), qui pourrait bien être l'hormone antimüllerienne retrouvée très élevée en cas de syndrome des ovaires polymicrokystiques.

\subsection{Comment faire le diagnostic de syndrome des ovaires polymicrokystiques?}

Rappelons que chez l'adulte, les critères retenus par la conférence de consensus de Rotterdam pour le diagnostic de syndrome des ovaires polymicrokystiques sont les suivants [2] :

- $\quad$ oligo- et/ou anovulation ;

- $\quad$ hyperandrogénie clinique et/ou biologique ;

- $\quad$ aspect échographique d'ovaires polymicrokystiques (présence de 12 follicules de 2 à $9 \mathrm{~mm}$ de diamètre dans chaque ovaire et/ou augmentation du volume ovarien supérieur à $10 \mathrm{~mL}$ ). Le seuil de 12 a été revu à 20 lors des recommandations de l'European Society of Human Reproduction and Embryology (Eshre) en 2018 [3].

Deux critères sur trois sont suffisants pour retenir le diagnostic de syndrome des ovaires polymicrokystiques, après exclusion des autres étiologies d'hyperandrogénie et/ou de dysovulation.

Nous détaillerons dans ce chapitre les critères échographiques permettant de diagnostiquer la présence d'ovaires polymicrokystiques.

\section{3. Échographie bidimensionnelle}

\subsubsection{Aspects techniques et recommandations}

La voie suspubienne doit toujours être la première étape de l'examen échographique puisqu'elle permet d'éliminer une anomalie utérine ou une pathologie ovarienne à développement abdominal ; elle permet également d'apprécier d'un coup d'œil la taille, la forme et la position des ovaires, ce qui peut guider la voie vaginale, lorsqu'ils sont en situation haute ou latérale en fosse iliaque. Elle sera bien sûr suivie d'un examen par voie transvaginale sauf chez les patientes vierges ou refusant l'examen. La voie transvaginale avec une sonde à haute fréquence (supérieure à $6 \mathrm{MHz}$ ) offre une bien meilleure résolution spatiale puisque les ovaires sont proches de la sonde vaginale et que le tissu graisseux est en principe moins gênant, sauf en cas de lipomatose pelvienne importante. Avec cette technique, non seulement la taille et la forme des ovaires, mais aussi leur structure interne, à savoir les follicules et le stroma, sont bien visualisables. 
Cependant, l'évaluation précise de la taille des ovaires n'est pas aisée et nécessite de choisir méticuleusement l'image sur laquelle l'ovaire apparaît le plus long et le plus large. L'image doit être gelée puis la surface calculée soit par l'utilisation d'une ellipse, soit en délimitant l'ovaire manuellement, la surface étant dans les deux cas calculée par l'appareil (Fig 1). La seconde méthode est plus fastidieuse mais préférable lorsque l'ovaire n'est pas de forme ellipsoïdale, il est aussi possible d'évaluer la surface ovarienne par la formule : longueur $(\mathrm{L}) \times$ largeur $(\mathrm{l}) \times 0,8$. Le volume permet une approche la plus complète et peut être estimé à partir des trois mesures de l'ovaire en utilisant la formule de l'ellipse $: \mathrm{L} \times 1 \times$ épaisseur $\times 0,523$ [4]. Cependant, les mesures doivent être prises dans trois plans orthogonaux stricts, ce qui n'est pas toujours respecté.

Le comptage du nombre total de follicules et l'évaluation de leur taille nécessitent de visualiser l'ovaire dans sa totalité en le balayant d'avant en arrière par exemple.

\subsubsection{Définition consensuelle des ovaires polymicrokystiques}

La définition des ovaires polykystiques déterminées lors du consensus de Rotterdam de 2003 est la suivante : au moins 12 follicules de 2 à $9 \mathrm{~mm}$ de diamètre par ovaire et/ou un volume ovarien augmenté (supérieur à $10 \mathrm{~cm}^{3}$ ) [5]. Le seuil de $10 \mathrm{~cm}^{3}$ pour discriminer un ovaire normal d'un ovaire polymicrokystique reste inchangé [5].

En ce qui concerne l'excès folliculaire, avec l'amélioration des performances des appareils d'échographie, il est désormais possible de mieux distinguer les petits follicules et notamment les follicules de moins de $2 \mathrm{~mm}$ de diamètre (fig. 2), ce qui rend le seuil de 12 follicules par ovaire obsolète à l'heure actuelle. Dans une revue de la littérature récente, le nombre de follicules antraux retenu pour parler d'ovaire polymicrokystique est désormais compris entre 19 et 25 follicules par ovaire lorsque l'appareil d'échographie est récent et que la fréquence maximale de la sonde endovaginale est supérieure à $8 \mathrm{MHz}$ [6]. L'Eshre recommande donc depuis 2018 d'utiliser le seuil de 20 follicules par ovaire en utilisant les sondes supérieures à $8 \mathrm{MHz}$ [3].

En cas d'impossibilité de réalisation d'une échographie fiable, le dosage de l'hormone antimüllérienne peut remplacer la mesure de la richesse en follicules. La concentration sérique d'hormone antimüllerienne, sécrétée par les petits follicules antraux, est en effet très bien corrélée avec le nombre de follicules de moins de $9 \mathrm{~mm}$ visibles en échographie et nous proposons un seuil de $4,2 \mathrm{ng} / \mathrm{ml}$ ou $30 \mathrm{pmol} / \mathrm{l}$ pour évoquer des ovaires polymicrokystiques avec son dosage automatisé [7]. 


\subsection{Autres critères diagnostiques}

\subsubsection{Surface ovarienne}

La surface ovarienne n'est pas retenue dans le consensus, mais dans notre expérience, son pouvoir diagnostique est en fait légèrement supérieur à celui du volume ovarien pour un seuil de $5,5 \mathrm{~cm}^{2}$ par ovaire.

L'hypertrophie stromale est caractérisée par une augmentation de la partie centrale de l'ovaire qui semble d'autre part plus échogène. Cependant l'appréciation du stroma est très subjective, et sa quantification nécessite le recours à des appareils d'échographie permettant des mesures et des calculs sophistiqués pour être exploitables de façon objective [8-10]. L'hypertrophie stromale n'est donc pas retenue dans la définition consensuelle des ovaires polymicrokystiques.

La distribution en périphérie des follicules a, elle aussi, été jugée comme trop inconstante et subjective pour être retenue dans la définition consensuelle.

\subsection{2. Échographie tridimensionnelle}

Pour éviter les difficultés de mesure de l'ovaire, l'échographie tridimensionnelle a été proposée. À partir des données stockées, trois plans octogonaux sont déterminés dans l'ovaire, permettant une évaluation plus précise des trois dimensions et ainsi du volume ovarien. Le comptage folliculaire peut se faire manuellement grâce mode triplan en balayant l'ovaire manuellement selon les trois plans orthogonaux ou grâce au mode multiplans TUI.

Il peut être également réalisé de façon automatique ou semi-automatique grâce au mode sono automatic volume calculation (AVC) qui permet une estimation du nombre, des dimensions et du volume individuel et global des follicules (fig. 3a). Ce mode est de réalisation facile et permet un comptage rapide des follicules notamment quand ils sont très nombreux,(fig $3 b$ ). Cependant la méthode n'est pas parfaite car elle méconnait souvent des follicules de petite taille qu'il faut revalider (fig 3c) et à l'inverse elle peut englober plusieurs follicules contigus (fig 3d). La nécessité de cette validation «manuelle » a posteriori fait de l'échographie tridimensionnelle un outil peu compatible avec la pratique courante, dans l'état actuel de ses performances.

L'aspect micropolykystique est également bien visible en reconstruction tridimensionnelle avec mode surfacique et inversion pour bien dissocier les follicules du stroma. Nardo et al. ont trouvé de bonnes corrélations entre les mesures par échographies bi- et tridimensionnelle du volume ovarien et du comptage folliculaire [11]. En pratique courante, il ne semble cependant pas y avoir de supériorité de l'échographie tridimensionnelle sur la technique bidimensionnelle.

En somme, l'étude échographique de l' ovaire polymicrokystique est aujourd'hui considérée comme un outil diagnostique qui requiert les mêmes critères de qualité qu'un dosage biologique. Cela 
suppose que les résultats soient exprimés en variables quantitatives et non plus seulement descriptives. Elle s'insère à part entière dans les critères diagnostiques du syndrome des ovaires polymicrokystiques au même titre que l'oligoanovulation et l'hyperandrogénie clinique ou biologique. Cependant, elle ne doit être utilisée par le clinicien que si l'échographiste est suffisamment entraîné et reproductible dans ses résultats. En cas d'impossibilité de réalisation d'une échographie fiable, le dosage de l'hormone antimüllérienne peut remplacer la mesure de la richesse en follicules. L'hormone antimüllerienne, sécrétée par les petits follicules antraux, est en effet très bien corrélée avec le nombre de follicules de moins de $9 \mathrm{~mm}$ visibles en échographie.

\section{Insuffisance ovarienne prématurée}

L'insuffisance ovarienne prématurée est définie par la survenue avant 40 ans d'une aménorrhée persistant plus de 4 mois associée à une concentration de FSH supérieure à 25 UI/L sur deux dosages distincts réalisés à quelques semaines d'intervalle, et à des signes plus ou moins marqués d'hypoœstrogénie : bouffée vasomotrice, sécheresse vaginale, trouble de l'humeur, insomnie, asthénie [12]. Il s'agit d'un hypogonadisme hypergonadotrope. L'aménorrhée ne répond en principe pas au test aux progestatifs.

\section{1. Étiologie}

Trois grands mécanismes histologiques ont été décrits : l'anomalie de la formation du pool folliculaire, la déplétion folliculaire précoce et le blocage de maturation folliculaire. Soixante-quinze pour cent des insuffisances ovariennes prématurées sont étiquetées idiopathiques. Les étiologies connues les plus fréquentes sont : les dysgénésies gonadiques et notamment le syndrome de Turner, les anomalies liées à l'X, la mutation de FMR1 et les polyendocrinopathies auto-immunes. Cependant, un nombre accru de gènes éventuellement responsables d'une insuffisance ovarienne prématurée sont de plus en plus souvent systématiquement recherchés, diminuant ainsi le nombre de cas idiopathiques. Dans une étude qui regroupait 357 cas d'insuffisance ovarienne prématurée à caryotype normal, il y avait chez $14 \%$ une histoire familiale d'insuffisance ovarienne prématurée et chez $14 \%$ des signes cliniques ou biologiques d'auto-immunité [13].

\subsection{Diagnostic}

L' insuffisance ovarienne prématurée est due à une perte folliculaire prématurée. Elle peut survenir avant la puberté, donnant alors un tableau d'aménorrhée primaire avec impubérisme. Si elle survient après la puberté, l'épuisement est le plus souvent progressif et le diagnostic peut être évoqué dès les premiers signes d'épuisement folliculaire marqué par le raccourcissement des cycles, une concentration d'hormone antimüllerienne effondrée et une concentration de FSH élevée (supérieure à 12 UI/L). Deux tiers des patientes atteintes d'insuffisance ovarienne prématurée avaient une puberté normale avec aménorrhée secondaire [17]. Seulement $25 \%$ des patientes avaient une aménorrhée 
secondaire brutale [14]. La fluctuation de la fonction ovarienne avec reprise spontanée et intermittente des cycles avant l'arrêt définitif, ainsi que l'impact psychologique d'un tel diagnostic doivent faire éviter le terme de ménopause précoce [15]. Une grossesse spontanée est rare (dans 1 à $5 \%$ des cas) mais possible.

Il s'agit d'un diagnostic non exceptionnel qui concernerait $1 \%$ des femmes de moins de 40 ans et $0,1 \%$ des femmes de moins de 30 ans [16].

\section{3. Échographie et dosage d'hormone antimüllerienne}

Chez l'adolescente, en cas d'aménorrhée primaire, après avoir éliminé une aplasie utérovaginale, l'insuffisance ovarienne peut révéler une dysgénésie gonadique (syndrome de Turner, dysgénésie gonadique pure, etc.). Les ovaires ne sont pas visibles à l'échographie et se réduisent le plus souvent à de simples bandelettes fibreuses (fig. 4). L'utérus est présent mais hypoplasique avec un endomètre atrophique et un Doppler utérin à haute résistance. L'étude de l'appareil urinaire doit être systématique pour rechercher des malformations rénales - rein en fer à cheval en particulier - fréquent dans le syndrome de Turner. Les radiographies peuvent montrer des signes associés au niveau des mains (brachymétacarpie du quatrième rayon) et des genoux (crochet tibial). Enfin, le diagnostic sera confirmé par le caryotype.

Chez la femme en période d'activité génitale, chez qui une insuffisance ovarienne prématurée est suspectée (aménorrhée secondaire, raccourcissement des cycles, résistance aux gonadotrophines), l'échographie et le dosage d'hormone antimüllerienne sont les examens de choix pour apprécier l'épuisement du stock folliculaire. Les fluctuations de la concentration de FSH rendent en effet l'utilisation de ce dosage plus aléatoire pour en faire un dépistage précoce [17].

L'échographie doit être réalisée en début de cycle (de j2 à j5); elle doit préciser la taille des ovaires et s'attacher à un comptage minutieux des petits follicules antraux de moins de $10 \mathrm{~mm}$, qui sont le reflet de la réserve folliculaire ovarienne (fig. 5a). Les valeurs seuil de ce compte des follicules antraux ne sont cependant pas consensuelles en ce qui concerne la baisse de la réserve ovarienne [18]. L'établissement de ces seuils est d'ailleurs de plus en plus difficile en raison de l'amélioration constante de la résolution des appareils échographiques qui permettent de mieux distinguer les microfollicules, en particulier les plus petits (de 1 à $3 \mathrm{~mm}$ ) (fig. 5b). Une attention particulière doit en outre être portée à ces petits follicules (inférieurs à $7 \mathrm{~mm}$ ), qui sont retrouvés corrélés négativement avec l'âge de façon significative, alors que le nombre de follicules de 7 à $10 \mathrm{~mm}$ resterait pratiquement stable jusqu'à 45 ans [19]. Un compte inférieur ou égal à 5-7 follicules pour les deux ovaires a été proposé comme prédictif d'une réponse insuffisante à la stimulation ovarienne (critères de Bologne), mais est de plus en plus débattu [20]. 
La mesure du volume ovarien comme marqueur de baisse de la réserve ovarienne peut également être utilisée. L'étude d'une population témoin de 57 femmes dans notre centre a retrouvé une limite inférieure de $3 \mathrm{~cm}^{3}$ pour le volume ovarien moyen et de $2,5 \mathrm{~cm}^{2}$ pour la surface [21]. Les patientes présentant des valeurs inférieures pourraient être suspectes de présenter une insuffisance ovarienne débutante. Bowen et al. ont rapporté une corrélation significative entre la diminution des dimensions ovariennes, l'augmentation de l'âge et des taux de FSH sériques [22]. Cependant, pour ce qui concerne l'aide à la décision en stimulation ovarienne, Elgindy et al. ne retrouvaient pas de différences du volume ovarien moyen entre les femmes de moins de 38 ans ayant une réponse ovarienne normale ou diminuée après stimulation $\left(4,1 \pm 0,66\right.$ contre $\left.3,4 \pm 0,71 \mathrm{~cm}^{3}\right)$ [23]. En outre, une revue réalisée par Broekmans et al. incluant dix études sur le volume ovarien a conclu à une faible utilité clinique de ce marqueur de la réserve ovarienne pour prédire une mauvaise réponse ovarienne [24]. Les résultats de l'étude plus récente de Jayaprakasan et al. ont confirmé ces données [25].

Le dosage d'hormone antimüllerienne, tout à fait bien corrélé avec le compte folliculaire, est lui aussi un très bon marqueur précoce d'insuffisance ovarienne débutante. La reproductibilité de son dosage d'un cycle à l'autre et au cours d'un même cycle en fait un marqueur de choix pour l'évaluation de la réserve ovarienne. La production d'hormone antimüllerienne décline avec l'âge et une concentration sérique diminuée est souvent témoin d'une réserve folliculaire défaillante. Une hormone antimüllerienne indétectable est la règle en cas d'insuffisance ovarienne prématurée installée.

En somme, l'insuffisance ovarienne prématurée est une cause non exceptionnelle d'aménorrhée de la femme jeune. L'étiologie est souvent difficile à préciser en dehors du syndrome de Turner.

L'apport de l'échographie est incontestable pour déceler les insuffisances ovariennes débutantes et adapter la prise en charge en aide médicale à la procréation en cas de souhait de grossesse.

Une préservation de la fertilité pourra être proposée à des femmes jeunes sans projet de grossesse immédiat, ayant une baisse de leur réserve ovarienne

\section{Références}

[1] Norman RJ, Dewailly D, Legro RS, Hickey TE. Polycystic ovary syndrome. Lancet 2007;370:685697.

[2] Rotterdam ESHRE/ASRM-Sponsored PCOS consensus workshop group. Revised 2003 consensus on diagnostic criteria and long-term health risks related to polycystic ovary syndrome (PCOS). Hum Reprod 2004;19:41-47. 
[3] Teede HJ, Misso ML, Costello MF, Dokras A, Laven J, Moran L et al. Recommendations from the international evidence-based guideline for the assessment and management of polycystic ovary syndrome. Hum Reprod. 2018;33(9):1602-1618.

[4] Sample WF, Lippe BM, Gyepes MT. Gray-scale ultrasonography of the normal female pelvis. Radiology 1977;125:477-483.

[5] Balen AH, Laven JS, Tan SL, Dewailly D. Ultrasound assessment of the polycystic ovary:international consensus definitions. Hum Reprod Update 2003;9:505-514.

[6] Dewailly D, Lujan ME, Carmina E, Cedars MI, Laven J, Norman RJ, Escobar-Morreale HF, Definition and significance of polycystic ovarian morphology: a task force report from the Androgen Excess and Polycystic Ovary Syndrome Society. Hum Reprod Update 2014;20(3):334-52.

[7] Pigny P, Gorisse E, Ghulam A, Robin G, Catteau-Jonard S, Duhamel A, Dewailly D. Comparative assessment of five serum antimüllerian hormone assays for the diagnosis of polycystic ovary syndrome. Fertil Steril 2016;105(4):1063-1069

[8] Al-Took S, Watkin K, Tulandi T, Tan SL. Ovarian stromal echogenicity in women with clomiphene citrate-sensitive and clomiphene citrate-resistant polycystic ovary syndrome. Fertil Steril 1999;71:952-954.

[9] Fulghesu AM, Ciampelli M, Belosi C, Apa R, Pavone V, Lanzone A. A new ultrasound criterion for the diagnosis of polycystic ovary syndrome:the ovarian stroma/total area ratio. Fertil Steril 2001;76:326-331.

[10] Pache TD, Hop WC, Wladimiroff JW, Schipper J, Fauser BC. Transvaginal sonography and abnormal ovarian appearance in menstrual cycle disturbances. Ultrasound Med Biol 1991;17:589-593.

[11] Nardo LG, Buckett WM, Khullar V. Determination of the best-fitting ultrasound formulaic method for ovarian volume measurement in women with polycystic ovary syndrome. Fertil Steril 2003;79:632-633.

[12] Goswami D, Conway GS. Premature ovarian failure. Hum Reprod Update 2005;11:391-410.

[13] Bachelot A, Rouxel A, Massin N, Dulon J, Courtillot C, Matuchansky C et al. Phenotyping and genetic studies of 357 consecutive patients presenting with premature ovarian failure. Eur J Endocrinol 2009;161:179-187.

[14] Christin-Maître S, Pasquier M, Donadille B, Bouchard P. L'insuffisance ovarienne prématurée. Ann Endocrinol (Paris) 2006;67:557-566. 
[15] Nelson LM, Covington SN, Rebar RW. An update:spontaneous premature ovarian failure is not an early menopause. Fertil Steril 2005;83:1327-1332.

[16] Coulam CB, Adamson SC, Annegers JF. Incidence of premature ovarian failure. Obstet Gynecol 1986;67:604-606.

[17] Klinkert ER, Broekmans FJ, Looman CW, Habbema JD, te Velde ER. The antral follicle count is a better marker than basal follicle-stimulating hormone for the selection of older patients with acceptable pregnancy prospects after in vitro fertilization. Fertil Steril 2005;83:811-814.

[18] Knauff EA, Eijkemans MJ, Lambalk CB, ten Kate-Booij MJ, Hoek A, Beerendonk CC et al. Antimullerian hormone, inhibin B, and antral follicle count in young women with ovarian failure. $\mathrm{J}$ Clin Endocrinol Metab 2009;94:786-792.

[19] Haadsma ML, Bukman A, Groen H, Roeloffzen EM, Groenewoud ER, Heineman MJ, Hoek A. The number of small antral follicles $(2-6 \mathrm{~mm})$ determines the outcome of endocrine ovarian reserve tests in a subfertile population. Hum Reprod 2007;22:1925-1931.

[20] Ferraretti AP, La Marca A, Fauser BC, Tarlatzis B, Nargund G, Gianaroli L. ESHRE consensus on the definition of 'poor response' to ovarian stimulation for in vitro fertilization: the Bologna criteria. ESHRE working group on POR definition. Hum Reprod 2011;26(7):1616-24.

[21] Jonard S, Robert Y, Cortet-Rudelli C, Pigny P, Decanter C, Dewailly D. Ultrasound examination of polycystic ovaries:is it worth counting the follicles ? Hum Reprod 2003 ;18:598-603.

[22] Bowen S, Norian J, Santoro N, Pal L. Simple tools for assessment of ovarian reserve (OR): individual ovarian dimensions are reliable predictors of OR. Fertil Steril 2007;88:390-395.

[23] Elgindy EA, El-Haieg DO, El-Sebaey A. Antimullerian hormone: correlation of early follicular, ovulatory and midluteal levels with ovarian response and cycle outcome in intracytoplasmic sperm injection patients. Fertil Steril 2008;89:1670-1676.

[24] Broekmans FJ, Kwee J, Hendriks DJ, Mol BW, Lambalk CB. A systematic review of tests predicting ovarian reserve and IVF outcome. Hum Reprod Update 2006;12:685-718.

[25]. Jayaprakasan K1, Campbell B, Hopkisson J, Johnson I, Raine-Fenning N. A prospective, comparative analysis of antimüllerian hormone, inhibin-B, and three-dimensional ultrasound determinants of ovarian reserve in the prediction of poor response to controlled ovarian stimulation. Fertil Steril. 2010;93(3):855-64. 


\section{Légendes des figures}

Figure 1. Échographie montrant un ovaire polykystique. a : mesure de la surface ovarienne par l'ellipse électronique ; b : mesure de la surface ovarienne par la mesure directe des deux axes orthogonaux.

Figure 2. Aspect échographique d'ovaires micropolykystiques : présence d'une vingtaine de microfollicules, dont certains sont inférieurs à $2 \mathrm{~mm}$. a : ovaire droit ; b : ovaire gauche.

Figure 3. Comptage folliculaire par échographie tridimensionnelle en mode sono automatic volume calculation (sono AVC). a : ovaire polymicrokystique, mode sono AVC, chaque microfollicule est délinéé automatiquement et matérialisé par une couleur propre la reconstruction volumique en bas à droite dénombre une trentaine de follicules dans l'ovaire ; b : aspect polymicrokystique majeur en mode sono AVC avec plus de 50 microfollicules en périphérie de l'ovaire ; c : mode sono AVC, le comptage folliculaire est imparfait et méconnait un certain nombre de petits follicules qu'il faut revalider manuellement (flèches), malgré cela l'aspect polymicrokystique est évident avec environ 50 follicules dans l'ovaire; $d$ : la délinéation englobe quatre microfollicules contigus (flèches), mais là encore l'aspect polymicrokystique est évident.

Figure 4. Dysgénésie gonadique (aménorrhée primaire). Cœlioscopie mettant en évidence une petite bandelette gonadique blanchâtre sans ovaire identifiable.

Figure 5. Insuffisance ovarienne prématurée. a : petits ovaires hypovascularisés, sans aucun follicule visible chez une patiente de 37 ans ; b : échographie à j4 chez une patiente de 39 ans ayant des cycles de 24 jours : ovaires paucifolliculaires, avec deux follicules à droite de 11 et $4 \mathrm{~mm}$ et trois follicules à gauche dont un de taille millimétrique. 


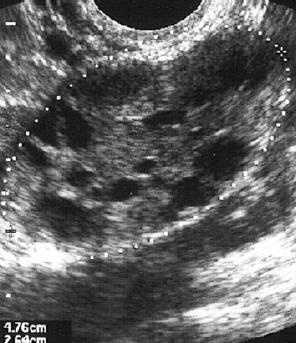




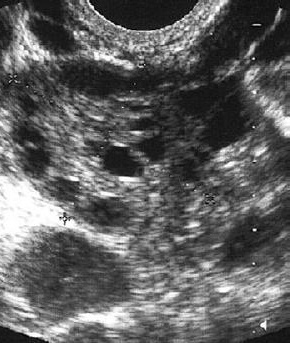




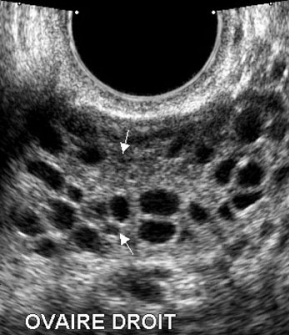




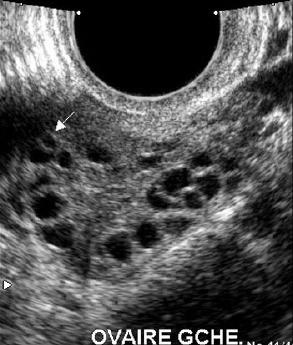




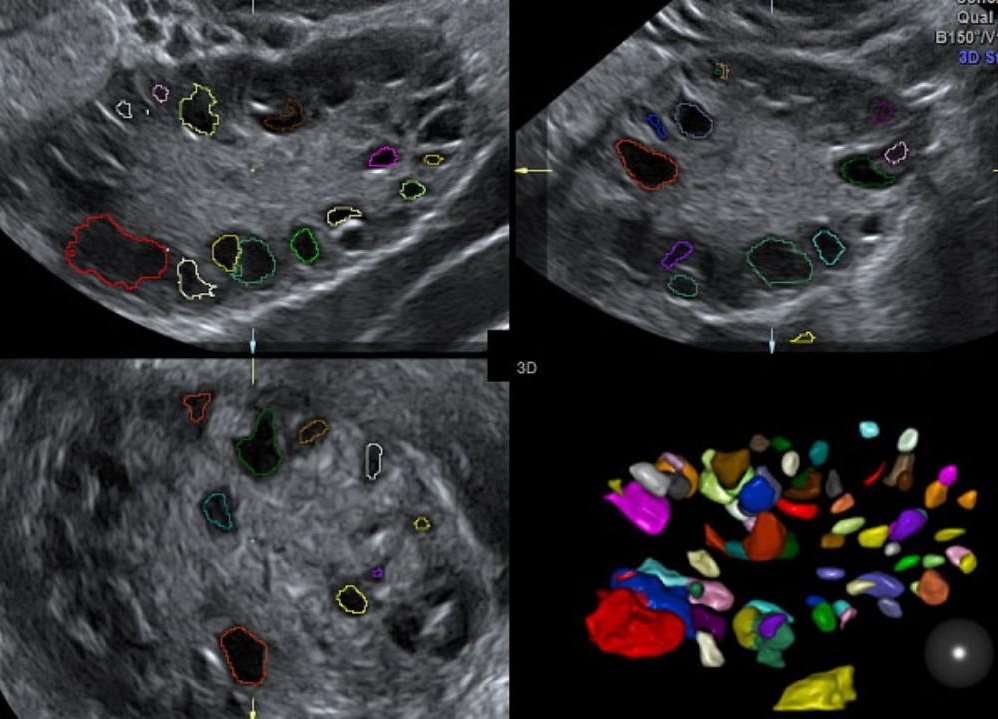



$+2$

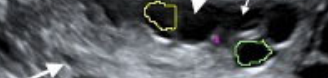

$\mathrm{B} 133^{\circ} / \mathrm{N} 120$
$\mathrm{CRI}$

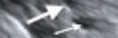

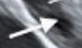

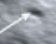
5

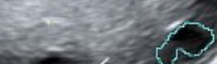

cone.
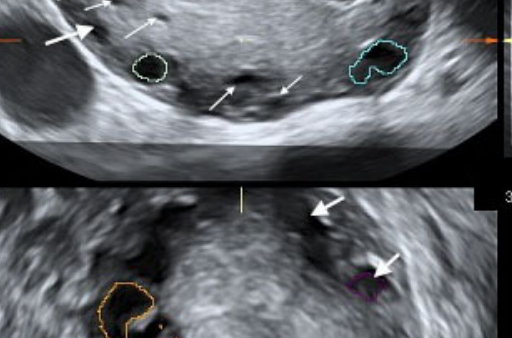

20
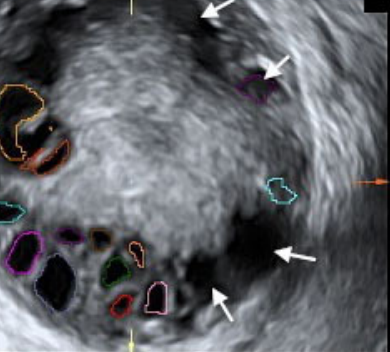
Stati

$3 D$

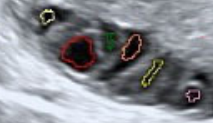

ify
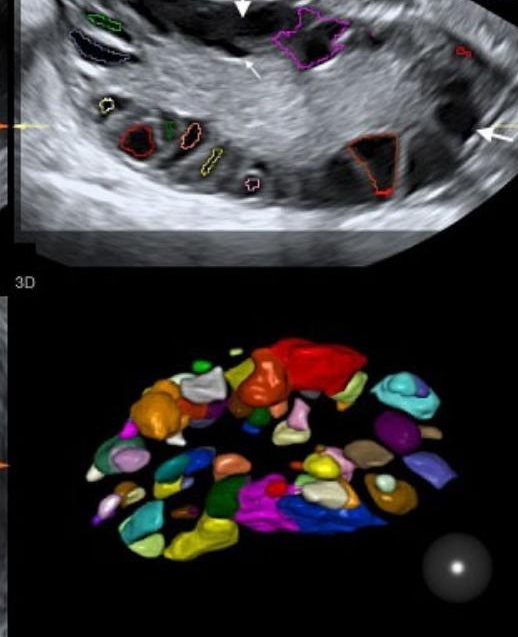

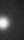



$\lim _{4} \mid$

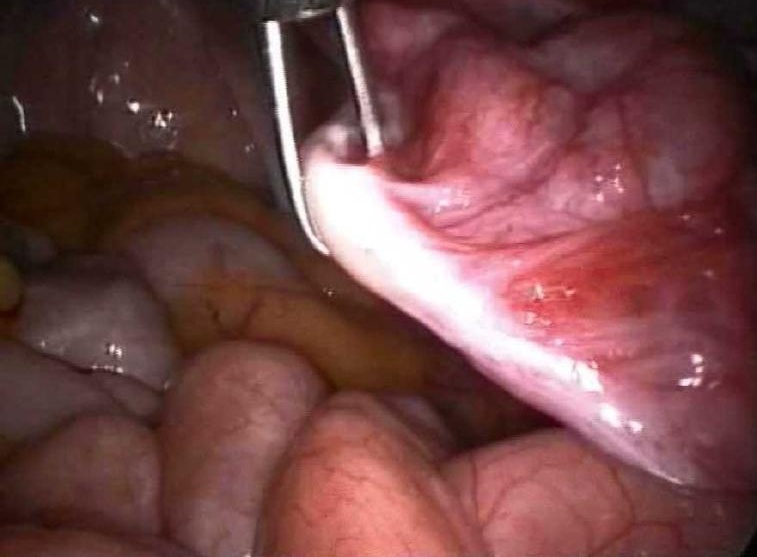




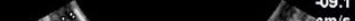

$2.9 . \% .6 \% \mathrm{~cm} / \mathrm{s}$

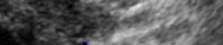

OVAIRE DROIT

\section{OVAIRE GCHE.}

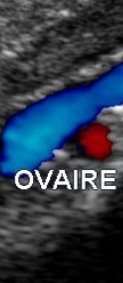


